\title{
Audit of Eye Care for Ventilated Patients in Intensive Treatment Unit During COVID-19 Pandemic
}

\author{
Muditha Dhanapala ${ }^{1 *}$, Suganya Sabaretnam ${ }^{2}$ \\ Senior Clinical Fellow ${ }^{1}$, Locum Consultant in Anaesthetics \& Intensive Care ${ }^{2}$, Luton and Dunstable \\ University Hospital, Bedfordshire Hospitals NHS Foundation Trust, United Kingdom
}

\begin{abstract}
Introduction
The protective ocular mechanisms are disrupted due to sedation and neuromuscular relaxants. This increases the risk of developing ocular surface disease (OSD) leading to increased post critical care morbidity. The risks are further increased in patients who are nursed prone as access to eyes is limited. Our aim was to determine whether eye care met the standard of the Ophthalmic Services Guidance and to improve the quality of care provided if found to be deficient.
\end{abstract}

\begin{abstract}
Methodology and Results
A prospective audit of the eye care provided to ventilated patients in critical care was done in April, 2020. An ophthalmological examination including fluoresce in staining was done to ascertain the prevalence of ocular morbidity present. Twenty-seven patients met the inclusion criteria and $88 \%$ patients were ventilated secondary to COVID-19 pneumonia. $35 \%$ of them were nursed prone at time of assessment; $81 \%$ had lagophthalmos (incomplete eye closure) while $53 \%$ of patients had a variant of OSD, including $23 \%$ with swollen conjunctival prolapse, $18 \%$ with conjunctivitis and $12 \%$ with corneal abrasions. None of the patients with lagophthalmos had received eye lubricants. Of the proned patients $50 \%$ had their eyes taped closed but only $33 \%$ had eye lubrication applied. We introduced local best practice guidance adapted from the Ophthalmic Services guidance. Education of critical care staff was undertaken and simplified guidance was exhibited in clinical areas.

Post-intervention audit was carried out against the standard after a one-month interval and 24 patients were included (80\% COVID-19 pneumonia); $67 \%$ had eye lubrication and $50 \%$ had eyes taped as per guidance in supine position. $100 \%$ of proned patients had eye lubricants with micropore taping.

Conclusion

During the COVID-19 pandemic, staffs were recruited from diverse backgrounds including theatre scrub nurses, recovery practitioners and operation department practitioners, to work alongside critical care nurses caring for ventilated patients. Education on the best ophthalmic practice guidance enabled holistic care to be provided to many ventilated patients by staff with limited prior experience in eye care in critical care.
\end{abstract}

Keywords: ocular surface disease, intensive care, eye care, COVID-19, Ophthalmic Services guidance

\section{Introduction}

Eye care is a significant aspect in the management of intensive care patients. The

*Correspondence: Muditha Dhanapala

E mail: dhanapalamuditha@yahoo.com

https://orchid.org/0000-0003-0044-6494

Received: 01/03/2021

Accepted: $17 / 04 / 2021$

DOI: http:/doi.org/10.4038/slja.v29i1.8755 protective ocular mechanisms are disrupted in this vulnerable group of patients, thus increasing the risk of developing ocular surface disease. OSD can present as direct corneal injury, exposure keratopathy, chemosis or conjunctivitis and keratitis. It leads to increased morbidity and requires further follow-up after recovering from the initial insult that required intensive care management. ${ }^{1}$

All mechanisms which protect the cornea and the contents of the orbital cavity are impaired in the ventilated patient. Sedation causes loss of the 
blink reflex and incomplete eye closure (lagophthalmos). The effect is worsened with the use of muscle relaxants as they cause further reduction of the tonic contraction of orbicularis oculi. The risk of direct eye compression, severe oedema, prolapse of swollen conjunctiva and chemosis are further increased in patients who are nursed prone. ${ }^{2}$ Restricted access to the eyes when prone also reduces quality of eye care. Further, in critically ill patients, treatment is often focussed on the management of organ failures, and eye care can become a side issue that may be unintentionally omitted. ${ }^{3}$ Therefore, protocolised eye care became essential during the current COVID-19 pandemic when all hospitals have seen great increases in the number of patients requiring ventilation and proning manoeuvres. Also, critical care areas were expanded with recruiting staff from various non-critical care background requiring up-skilling programs.

Ophthalmic services guidance for eye care in the intensive care unit was published by the Royal college of Ophthalmologists in collaboration with Faculty of intensive care medicine and Intensive Care Society (ICS) in 2017. It intends to educate intensive care staff on assessment of common eye injuries in critically ill patients and recommends in-cooperating eye assessment to the routine intensive care assessment and managing it accordingly. Further, it has emphasized the requirement of a clear unit-based protocol for assessment and intervention to minimize ocular problems and referring to the ophthalmology team if either a serious eye condition is suspected or no improvement in 48 hours following guided treatment. ${ }^{1}$

Corneal abrasions in most cases can be minimized by applying eye lubricant such as VitA POS or Xalin ointment while chloramphenicol eye ointment is used for conjunctivitis or red, sticky eyes. The patients with damaged cornea in untreated exposure keratopathy are highly vulnerable to having microbial keratitis and such deeper infection may lead to permanent and severe damage, even loss of vision. ${ }^{4}$

A study done by Hamadan University of Medical Sciences, Iran found that measures for eye care depended on the intensive care centre and including application of lubricants, chambers or eyelid closure. They identified that the most effective method to avoid exposure keratoplasty is by the application of polyethylene moisture in lower chamber. The study highlighted that adherence to simple protocol and education of intensive care staff about the eye care would improve the quality of eye care in critically ill patients. $^{4}$

Around $20-42 \%$ of critically ill patients suffer from exposure keratopathy and corneal abrasion, while $60 \%$ of those are sedated for more than two days due to impaired blink reflex. ${ }^{1}$ Such prolonged epithelial defects lead to scarring or even perforation of cornea in severe cases. Chemosis (conjunctiva bulges out due to conjunctival oedema) occurs in ventilated patients because of compromised venous return from the orbital structures, part of generalised oedema, gravitational forces of increased hydrostatic pressure, increased capillary leak or incomplete eyelid closure in high risk group. ${ }^{4}$

Protective measures to prevent these injuries are lubrication if grade 1 exposure (eyes slightly open with some conjunctival exposure), lubrication, manual closure or taping the lids with micropore tape along the lash margin for grade 2 exposure (Cornea exposed), whereas no specific action is required in grade 0 (eye lids close well) exposure unless the patient is nursed in prone position whose eyes re-lubricating every 4 hours and taping shut is recommended. ${ }^{1,2}$

\section{Objectives}

The main objective was to assess the compliance of eye care given to ventilated patients in the intensive treatment unit matched with the standard and identify the possible ophthalmic condition by fluorescein staining collaborating with the ophthalmology unit. If care found to be deficient, we planned to figure out the additional risk factors, implement the best practice guidance, education of critical care doctors and nurses, and regular ophthalmic review by the trust ophthalmology unit, especially during the COVID-19 pandemic period.

\section{Methodology}

The audit was a prospective cross-sectional single centre study. The ventilated patients cared at the intensive treatment unit, Luton and Dunstable hospital over the month of April 2020 were recruited for the first audit cycle. We excluded the patients who are not sedated and have the capacity to protect their eyes with blink reflex (patients on tracheotomies). The data was 
collected by the investigators performing bed side ocular examination, nursing records and drug prescription charts. Post-implementation audit was carried out over the month of June adhering to the same standard in the same clinical area.

\section{Results of the first audit}

The number of patients admitted to the ITU during pre-intervention period was 45 , and six of them were self-ventilating and had tracheotomy. Twenty-seven patients were ventilated under sedation, and 23 patients were nursed in prone position at some point after admission. The age of the patients included in the study ranged from 31 to 80 years with the mean age being 47 years and male predominated $(80 \%)$ in admission to the unit.

Majority of the patients were ventilated for type 1 respiratory failure secondary to COVID-19 infection (88\%) while $12 \%$ were for non COVID indications. Patients were ventilated for range of 1- 18 days with mean duration of being on ventilator was 6.4 days.

Two patients had pre-existing glaucoma and were on eye drops prior to the admission. Subsequently one of them was referred to ophthalmologist secondary to chemosis on both eyes.

On examination of grading of lagophthalmos, $66 \%$ had grade 1 exposure and $19 \%$ had grade 0 exposure. $15 \%$ of ventilated patients suffered from grade 2 exposure (Chart 1). However, none of the lagophthalmos were treated appropriately.

\section{Chart 1}

Grading of ocular examination

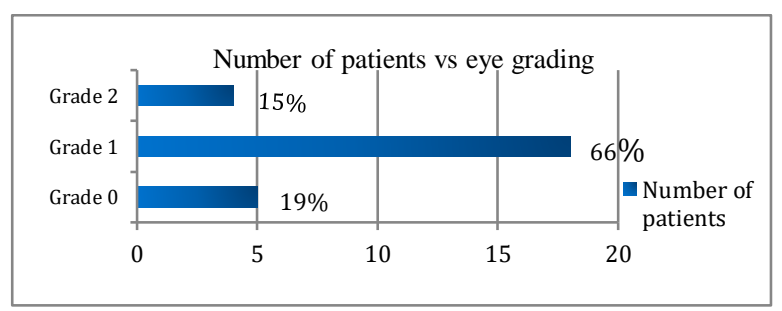

$83 \%$ of patients were nursed in prone position once or more times at some point after intubation and $35 \%$ of them $(n=9)$ were in prone position at the time of study. Fifty percent of them had their eyes closed with micropore taping, while 33\% had eye ointment utilized. Only one patient had both lids closure and the eye drops. Further analysis revealed that this patient was on regular eye treatment for glaucoma before admission to the unit. None of the patients has had their eye review in four-hour intervals.

Further, the ITU was expanded with recruiting health care staff from other specialities such as doctors, theatre scrub nurses, recovery practitioners and operation department practitioners after several training sessions in order to combat the increased workload in COVID-19 pandemic. During the study period, $77 \%$ of the staff were from non-critical care background. The awareness of best practice guidance of eye care was minimal in the staff including the doctors.

\section{Implementation of measures}

Hence it was clearly evident that the compliance of eye care was below par, we introduced several strategies to improve the compliance.

We requested guidance from the trust ophthalmology unit. One of our ophthalmology consultant colleagues volunteered to offer his expertise to the intensive care. Fluorescein staining was done by him when indicated and relevant treatment for diagnosed eye conditions was prescribed (Chart 2). It was evident that $53 \%$ of patients suffered from at least one OSD.

\section{Chart 2}

Ophthalmic diseases found in the initial critical care visit by Consultant ophthalmologist

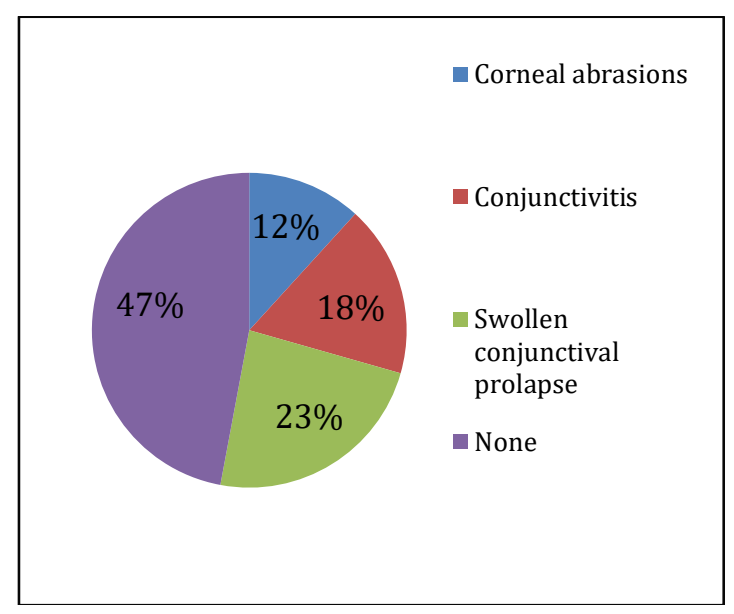

A simplified version of the best practice guidance was displayed at critical care area (Figure 1). ITU development nurse educated the staff from non-critical care background who had been redeployed to the intensive care during COVID-19 pandemic. Pharmacists ensured continued supply of eye lubricants. The consultant ophthalmologist reviewed patients twice a week and continued to support the 
intensive care team. Ophthalmology referrals were made for issues identified on other days. The pre-proned check list ensured all these patients receive eye lubrication and taping before turning.

\section{Figure 1}

The best practice guidance implemented in the intensive care setting

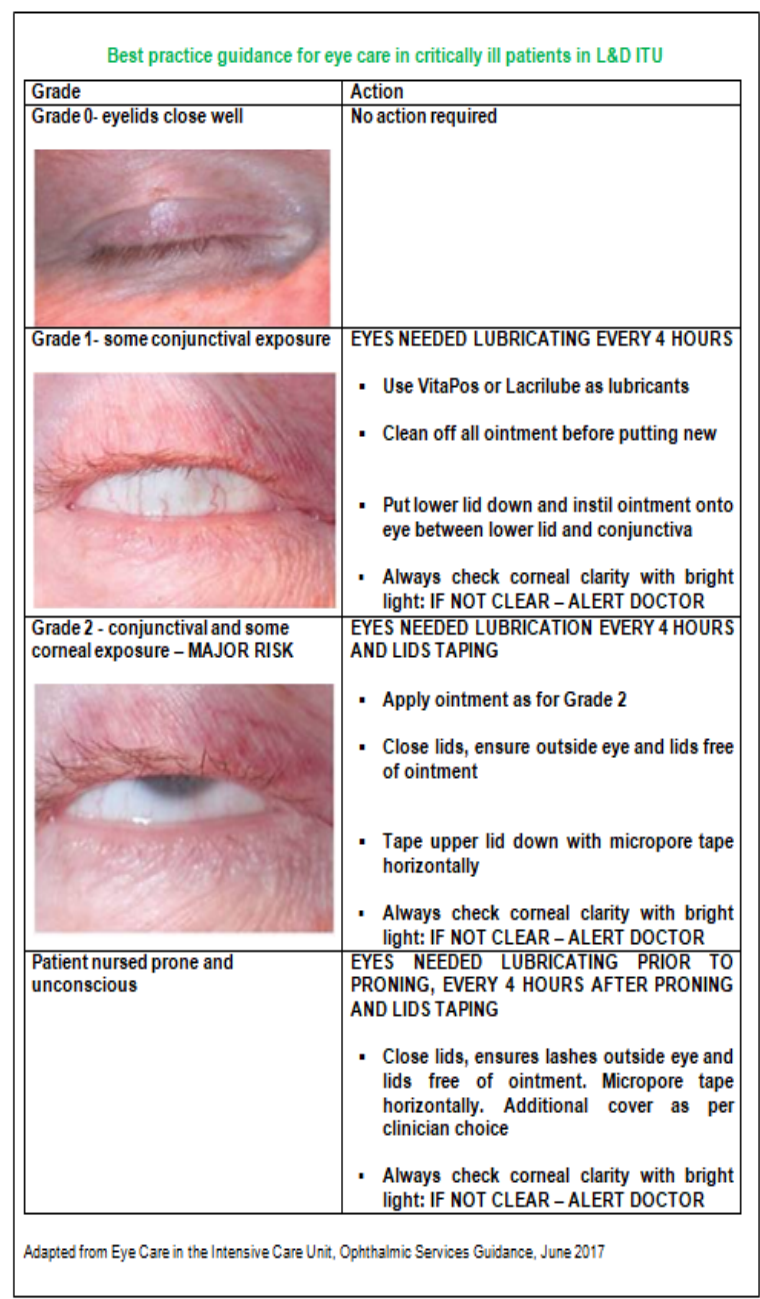

\section{Results of the follow-up audit}

The post-intervention study was carried out including 23 ventilated patients and $80 \%$ of them were ventilated for COVID-19 pneumonia. Five of them were in prone position at the times of data collection while $70 \%$ were proned at some point after admission. In ocular examination, $56 \%$ of patients were found to have lagophthalmos. Significant improvement from $0 \%$ to $67 \%$ was noted in eye lubrication for patients who had grade 1 and 2 exposure while $50 \%$ improvement in eye taping for grade 2 exposure. $100 \%$ of the patients in prone position had the eye lubricants with micropore taping prior to turning (Chart 3). The patients who suffered from the OSD made a good recovery with ophthalmology guided treatment.

\section{Chart 3}

Pre and post intervention of the best practice guidance in the intensive care setting

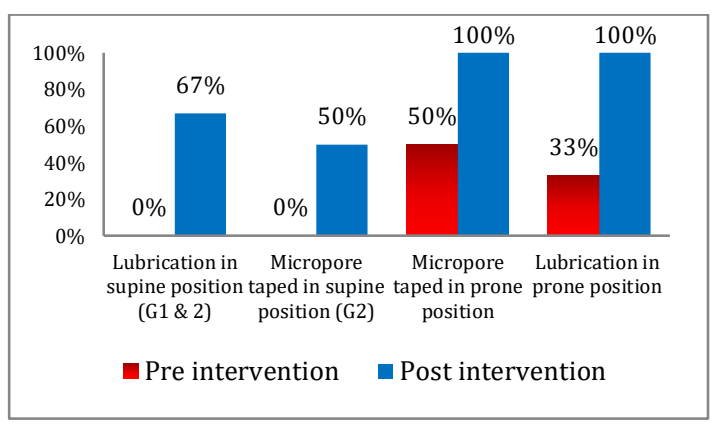

\section{Discussion}

The eye is a specialized highly vascular organ, which is susceptible to affected not only from underlying disease, but also due to exogenous process in critically ill patient. In ICU patients, presentation may vary from simple ocular condition to serious condition such as endophthalmitis, glaucoma and visual loss. Therefore, ocular examination should always be included in daily patient's examination. If the eye is neglected in vulnerable patients, the patient's quality of life may be compromised. ${ }^{5}$ In our audit, we encouraged the doctors to enter the grading of ocular examination in daily review notes.

The risk factors for OSD could be categorized under disease processes, treatment processes and ICU environment. The patients with pre-existing eye conditions or neurological deficits, on immunosuppressant, reduced GCS or fluid misdistribution are highly vulnerable to developing OSD. ${ }^{3}$ However, the major contribution for eye disease is associated with the specific treatment received by the patients such as mechanical positive pressure ventilation, usage of sedation or muscle relaxants in which the blink rate and reflex is significantly reduced increasing the risk of lagophthalmos. ${ }^{5}$ Prolong ITU stay, exposure to multi-resistant organism, and repeated tracheal suctioning have further enhanced the risk. ${ }^{6}$ Considering these facts, study population in this audit cycle had the multiple risk factors to develop OSD as nine out of ten patients were intubated for severe pneumonia where they had extended ventilator days, multiple positioning and suctioning manoeuvres and were on immunosuppressant depending on the different treatment trials. 
In those ventilated prone, can develop complicated eye injuries such as central retinal artery occlusion, bilateral acute glaucoma and ischemic optic neuropathy which may result even sudden visual loss. These are secondary to reduction of ocular perfusion pressure following raised intra-ocular or inta-orbital pressures with without concurrent systemic hypotension. ${ }^{7}$ Therefore, it is highlighted that eyes should be examined regularly even in the prone position. According to the best practice guidance, the patients in prone position benefit from eye lubrication and the micropore taping irrespective of their exposure of the eye. ${ }^{7}$ In our audit, best practice guidance together with the pre-prone checklist, improves eye care to be maintained to the standard that is recommended.

Adequate eye care includes frequent assessment from both physicians and nursing personnel, and should be considered the standard of care in the ICU. With the background of recruited personnel from varies specialties including the doctors and frequent rotation of staff, continuity of eye care was difficult. This issue was overcome with repeated educational program including bedside teaching. Finally, such programs will be carried out as required in the future to certify the continuity of best practice.

\section{Conclusion}

Education of staff caring for critically ill patients improves eye care in intensive care. Easily accessible simplified best practice guidance improves adherence to the standard care. As the circulation of staff from non-critical care specialities is increased during the COVID-19 pandemic, repeated education is needed.

\section{References}

1. Lightman S, Montgomery H. Ophthalmic Services Guidance, Eye care in the intensive care unit. 2017; 1-13.

https://www.ncbi.nlm.nih.gov/pmc/articles/PMC $\underline{6259085}$

2. Werli-Alvarenga A, Ercole FF et al. Corneal injuries: incidence and risk factors in the intensive care unit. Rev Lat Am Enfermagem. 2011; 19(5): 1088-95. https://pubmed.ncbi.nlm.nih.gov/22030572

3. Hearne BJ, Hearne GH, Montgomery $\mathrm{H}$, et al. Eye care in the intensive care unit. Journal of the Intensive Care Society. 2018; 19(4): 345-350. https://pubmed.ncbi.nlm.nih.gov/30515243

4. Zahra T-K. Eye care in the intensive care patients: evidence-based review. BMJ Open. 2017; 7: A1-A78. https://www.ncbi.nlm.nih.gov/pmc/articles/PMC 5759406

5. Ramirez F, Ibarra S, Varon J, et al. The neglected eye: ophthalmological issues in the intensive care unit. Critical Care Shock 2008; 11:72-82.

https://www.researchgate.net/publication/266869 $\underline{934}$

6. Rosenberg JB, Eisen LA. Eye care in the intensive care unit: narrative review and metaanalysis. Critical Care Med 2008; 36: 31513155.

https://pubmed.ncbi.nlm.nih.gov/18936706

7. Panchabhai TS, Bandyopadhyay D, Kapoor A, et al. Acute ischemic optic neuropathy with extended prone position ventilation in a lung transplant recipient. Int J Crit Illn Inj Sci 2016; 6: $45-47$. 